\title{
Revisión Sistemática de la Habilidad de Autocontrol del Estudiante y su Rendimiento Académico en la Vida Universitaria
}

\author{
Maria V. Pérez-Villalobos ${ }^{(1)}$, Rubia C. Cobo-Rendón ${ }^{(2)}$, Fabiola M. Sáez ${ }^{(2)}$ y Alejandro E. Díaz-Mujica ${ }^{(1)}$ \\ (1) Fac. de Ciencias Sociales, Dpto. de Psicología, Universidad de Concepción, Concepción-Chile. \\ (e-mail: marperez@udec.cl; adiazm@udec.cl) \\ (2) Fac. de Ciencias Sociales, Programa de Doctorado en Psicología, Universidad de Concepción, \\ Concepción-Chile (e-mail: rubiacobo@udec.cl; fabiosaez@udec.cl)
}

Recibido Oct. 18, 2017; Aceptado Dic. 5, 2017; Versión final Ene. 11, 2018, Publicado Jun. 2018

\begin{abstract}
Resumen
El presente trabajo tuvo tres objetivos: (1) identificar los diseños de investigación más empleados en estudios sobre la relación entre autocontrol y rendimiento académico en estudiantes universitarios, (2) presentar las conceptualizaciones de autocontrol y rendimiento académico en esos estudios, y (3) identificar el grado de asociación entre estas variables. Se realizó una revisión sistemática de las bases de datos Web of Science, Scopus, Scielo y Eric, con los descriptores "estudiantes universitarios, autocontrol y rendimiento académico". Se encontró un total de 173 artículos que muestran que los diseños de investigación predictivos-explicativos son los más empleados para el estudio de la relación entre autocontrol y rendimiento académico. Este trabajo permite concluir que existe una relación directa entre el autocontrol y el rendimiento académico, observable cuando se emplean medidas objetivas para el estudio de estas variables.
\end{abstract}

\section{A Systematic Review of the Student Self-Control Ability and Academic Performance in University Life}

\begin{abstract}
The present work had three objectives: (1) to identify the research designs most used in studies on the relationship between self-control and academic performance in university students, (2) to present the conceptualizations of self-control and academic performance in those studies and (3) to identify the degree of association between these variables. A systematic review of the literature was performed by reviewing the databases: Web of Science, Scopus, Scielo and Eric, with the descriptors "university students, self-control and academic performance". A total of 173 articles were found, articles that show that the predictiveexplanatory research designs are the most used for the study of the relationship between self-control and academic performance. The work shows that there is a direct relationship between self-control and academic performance, observable when objective measures are used to study these variables.
\end{abstract}

Keywords: systematic review; self-control; university students; academic performance 


\section{INTRODUCCIÓN}

Existe un creciente interés de investigación sobre aspectos que promueven el desarrollo óptimo de estudiantes universitarios en distintas latitudes, esto refleja el reconocimiento de que sus vidas son diferentes en muchos aspectos a los experimentados por los estudiantes en décadas anteriores (Conley, et. al., 2014). La vida universitaria es más compleja, el ingreso y su permanencia conlleva al aumento de niveles de ansiedad, estrés y depresión (Eckberg et al., 2017), sin contar con el costo emocional, económico y social que generan las dificultades para su integración. Se espera que las políticas y estrategias que las universidades implementen tengan un resultado favorable en la calidad de la educación y en la vida de los estudiantes. Las universidades requieren identificar y conocer cuáles aspectos en la vida de los jóvenes pueden ser mejorados para impactar de forma positiva contribuyendo a su desempeño académico, lo que hace necesario contar con instrumentos de medida fiables y validados que permitan examinar esos aspectos, y disponer de procedimientos que den respuesta a sus necesidades.

Reunir las evidencias obtenidas en estudios empíricos sobre un mismo problema, es uno de los aportes de la revisión sistemática en un determinado campo y, en este caso, en educación. Este tipo de metodología permite distinguir las mejores pruebas desarrolladas sobre un tema en específico (SánchezMeca y Botella, 2010). Se caracteriza por ser rigurosa en términos de criterios de inclusión y de calidad, tiene un carácter informativo al responder a preguntas claramente delimitadas y exhaustivas mediante el uso de la información disponible; se presenta con un diseño explicito basado en una descripción detallada para responder las preguntas planteadas (Vidal, et al. 2015). Las revisiones sistemáticas pueden clasificarse en dos tipos: (1) revisiones cuantitativas o meta-análisis, caracterizadas por el empleo de métodos estadísticos para analizar los datos obtenidos, y (2) revisiones "cualitativas" que abordan la información mediante análisis críticos. La relevancia de esta metodología está en su capacidad de proporcionar antecedentes para el avance científico en un tema de interés, la identificación de contradicciones, vacíos y desafíos emergentes. En el caso del contexto educativo del presente trabajo, el desarrollo de revisiones sistemáticas proporciona evidencias para ayudar a encontrar orientaciones y diseñar políticas de apoyo a estudiantes (Páramo y Hederich, 2014).

En la Educación Superior existe interés por el desarrollo de trabajos referidos no solo a la formación intelectual sino, también, al aprendizaje de competencias, habilidades emocionales y sociales (Tarbetsky et al., 2017). En este escenario, el autocontrol se ha convertido en un concepto prominente de la investigación en psicología y en sus distintas áreas, como educativa, social, clínica, neuropsicológica (de Ridder et al., 2012). Históricamente el autocontrol ha sido visto como una virtud maestra que hace posibles todas las demás virtudes, clave del éxito adaptativo y aspecto central para el comportamiento virtuoso (Baumeister y Exline, 1999). Ha tenido modificaciones en su definición a lo largo del tiempo, por ejemplo, fue entendido como parte de la teoría de la autorregulación, constituida por cuatro dominios: control de los pensamientos, emociones, impulsos y rendimiento. El control de pensamientos hace referencia a la autorregulación de la concentración, razonamiento, inferencia y supresión de pensamientos no deseados. El control de emociones se refiere a la regulación del estado de ánimo. El control de los impulsos alude a resistir y anular formas de actuar indeseables a nivel personal o social (ej. impulsos de acciones en exceso, impulsos violentos o agresivos) siendo fundamental para lograr un desempeño óptimo, incluyendo alta persistencia, gestión del esfuerzo y otros aspectos vinculados con el logro de objetivos (Baumeister y Exline, 2000).

El autocontrol fue considerado como la capacidad de anular, cambiar o interrumpir las respuestas internas (como impulsos) y abstenerse en función de ellas. Posteriormente, fue descrito como la capacidad de resistir las tentaciones, regular las emociones, cogniciones, controlar y ajustar el comportamiento en servicio de objetivos generales a largo plazo; alterando las propias responsabilidades con la finalidad de alinearlas con estándares tales como ideales, valores, moral y expectativas sociales, y así apoyar la búsqueda de objetivos a largo plazo (Baumeister, et al., 2007). A continuación, fue descrito como la capacidad de controlar impulsos y deseos a corto plazo que presentan conflictos con objetivos establecidos a largo plazo (Hofmann, et al., 2012). También ha sido visto como un estado (variaciones del autocontrol dependiendo de las situaciones y el tiempo) o como un rasgo (estado disposicional que es relativamente estable en las situaciones y el tiempo) (de Ridder et al., 2012). En tal sentido el autocontrol puede ser considerado como la capacidad para anular o modificar las respuestas internas, incluyendo impulsos, pensamientos, emociones y comportamientos presentes en situaciones a corto plazo con la finalidad de obtener otros beneficios a largo plazo que pueden estar en consonancia con estándares, valores o expectativas sociales que son consideradas relevantes para las personas. Este constructo incluye aspectos como planificar objetivos a largo plazo, el mantenimiento de comportamientos en pro de las metas, retrasar gratificaciones inmediatas, resistir las tentaciones y controlar pensamientos, impulsos y comportamientos. 
Es considerado un aspecto relevante para casi todas las formas de comportamiento, puesto que conduce a una vida satisfactoria, en que las personas con altos niveles de autocontrol presentan mayor satisfacción con sus vidas (Hofmann, et. al., 2014; Wiese, et al., 2017). Siendo vigente hoy en día la afirmación de que el autocontrol ayuda a las personas florecen dentro de la sociedad y permite que funcionen de manera más eficaz (Uziel y Baumeister, 2017). A nivel empírico, en el 2012 se realizó un metanálisis con el objetivo del examinar la evidencia sobre la relación entre autocontrol disposicional y diversos tipos de comportamiento, siendo un primer intento de integrar los resultados de estudios empíricos sobre el autocontrol que emplean diferentes diseños y diferentes poblaciones. En este caso informaron sobre resultados de 102 estudios (total $\mathrm{N}=32648$ ) que buscaban investigar los efectos del auto-control utilizando la escala de autocontrol de Tangney et al., 2004, la escala de Impulsividad de Barratt, y la escala de bajo auto-control de Grasmick et al., 1993, por ser las más referenciadas en la literatura. Se describe una relación entre los comportamientos deseados (control de peso, bienestar, rendimiento en la escuela o el trabajo) y la inhibición de los comportamientos no deseados, (atracones, consumo de alcohol), pero sus efectos varían claramente en los diferentes dominios de la vida (por ejemplo, el logro, ajuste) (de Ridder, et al., 2012). Otras revisiones sobre el autocontrol describen su importancia teórica, empírica y práctica, mostrando su relación con comportamientos deseados y el efecto de estos en distintas áreas de la vida (Duckworth y Kern, 2011; Burnette et al., 2014; Piquero et al., 2016; Vazsonyi et al., 2017).

En el contexto académico, el autocontrol es un constructo beneficioso para el ajuste académico (Duckworth et al., 2010), el control del esfuerzo, el manejo de la impulsividad (Valiente et al., 2013) y puede ser visto como un facilitador del afrontamiento a tareas que generan estrés (Finkenauer et al., 2005), lo que permite afirmar que los estudiantes con altos niveles de autocontrol son más autónomos y exitosos. Otros estudios refieren que las personas con alto autocontrol presentan mejores calificaciones, en comparación con las personas con bajo contenido de autocontrol. Por ejemplo Tangney, et al. (2004) realizaron dos estudios investigando la relación entre el autocontrol y múltiples variables, incluidos los grados de la Universidad (un primer estudio con 351 estudiantes de Psicología y otro con 255), reportaron relaciones directas entre el rendimiento académico (calificaciones) y el autocontrol. Los estudiantes con mayor autocontrol culminan a tiempo sus tareas logrando evitar que las actividades de ocio interfieran con el trabajo académico y permitiendo que el tiempo de estudio sea empleado de forma efectiva, evitando distracciones que perjudiquen el desempeño.

En años recientes Bertram, et al., (2016), desarrollaron un estudio con el objetivo de analizar el impacto del autocontrol disposicional, la autoeficacia y la autoestima en los problemas relacionados con la ansiedad en estudiantes de escuela secundaria $(n=158)$. Plantearon la hipótesis que estos tres rasgos de la personalidad tendrían una relación negativa con el deterioro de la cognición producido por ansiedad. Se esperaba que el autocontrol ayudaría a mitigar la reducción en la cognición debido a la ansiedad. Para ello los estudiantes completaron medidas de auto-control, autoeficacia y autoestima al comienzo del año escolar, cinco meses después los investigadores evaluaron el nivel de deterioro de la cognición debido a la ansiedad durante los exámenes de matemáticas. En los resultados describen que una mayor capacidad de autocontrol se relaciona con menor deterioro de la cognición debido a la ansiedad cinco meses más tarde. El autocontrol fue indirectamente relacionado con las calificaciones en matemáticas por medio de la disminución de la cognición debido a la ansiedad. Los hallazgos sugieren que la mejora de la capacidad de autocontrol puede permitir a los estudiantes para hacer frente a los problemas relacionados con la ansiedad durante los exámenes escolares. Estos antecedentes refuerzan la idea que el autocontrol es una característica por excelencia de las conductas de autorregulación (de Ridder et al., 2012).

El rendimiento académico, este puede ser definido como los resultados obtenidos por el estudiante a partir de las calificaciones obtenidas durante su carrera, medidos mediante las calificaciones en exámenes o pruebas (Pérez-Cárceles, et al., 2014). Es un concepto que está determinado por diversos aspectos como las características propias del estudiante (género, calificaciones anteriores, puntuaciones en pruebas de ingreso a la universidad) (Soria-Barreto y Zúñiga-Jara, 2014), o de aspectos externo como el tipo de diseño curricular implementado en la universidad (Avendaño, et al., 2016). Estudios metanalíticos han analizado su relación con otras variables, una investigación revisó 33 trabajos que relacionaban la procrastinación y el rendimiento en estudiantes de distintos niveles educativos. Los investigadores evaluaron el nivel de relación considerando el tipo de instrumentos empleados y el indicador de rendimiento académico utilizado, en estudiantes de primaria, secundaria y de etapa universitaria, entre los hallazgos más relevantes se encuentranReunir, relaciones negativas y significativas entre la procrastinación (considerada como falta de autorregulación) y el rendimiento académico $(r(38529)=-.16, p<0.01)$, estos autores refieren que la relación entre estas variables podría incrementarse o disminuirse dependiendo del indicador de rendimiento académico seleccionado y la escala de medida empleada para el estudio de la procrastinación (Kim y Seo, 2015). 
En base a los estudios anteriormente descritos, se ha demostrado que el autocontrol presenta múltiples beneficios a la vida académica (mayor ajuste, mayor esfuerzo y afrontamiento), específicamente hay indicios que el autocontrol tiene efectos positivos en el rendimiento académico, descrito en los estudios de meta-análisis e investigaciones específicas, sin embargo no queda claro los diseños mayormente empleados en su estudio. Considerando la amplitud en las conceptualizaciones del autocontrol se justifica la necesidad de conocer cuáles son las conceptualizaciones más empleadas en el contexto universitario y la magnitud de su relación con el rendimiento académico, en tal sentido la presente investigación tiene tres objetivos: (1) identificar los diseños de investigación más empleados en estudios sobre la relación entre autocontrol y rendimiento académico en estudiantes universitarios, (2) presentar las conceptualizaciones de autocontrol y rendimiento académico utilizadas en esos estudios y (3) identificar el grado de asociación encontrado entre estas variables.

\section{MÉTODO}

Se realizó una exploración electrónica y sistemática de artículos, mediante la consulta de los buscadores científicos Web of Science (http://www.webofknowledge.com), Scopus (https://www.scopus.com), Scielo (https://www.scielo.org) y Eric (https://eric.ed.gov/). Se tuvieron en cuenta los lineamientos para el desarrollo de revisiones científicas propuesto en el sistema Preferred Reporting Items for Systematic reviews and Meta-Analyses (PRISMA). El análisis de los estudios se realizó teniendo en cuenta los objetivos propuestos, se identificaron los diseños de investigación más empleados, las conceptualizaciones de autocontrol y rendimiento académico aplicadas en las investigaciones seleccionadas y se analizó la presentación del grado de asociación de estas variables.

\section{Criterios de inclusión, selección de los datos}

Para seleccionar los artículos, se emplearon los descriptores "estudiantes universitarios" (university students), "autocontrol" (self-control OR self control OR selfcontrol) y "rendimiento académico" (academic performance OR academic achievement OR student achievement OR student progress OR academic progress); se abarcaron todos los artículos publicados en idioma inglés y/o español en el periodo comprendido entre los años 2006 y 2017. Se consideraron artículos que estudiaran de forma cuantitativa al autocontrol y su relación estadística con el rendimiento académico en la vida universitaria. Se excluyeron los artículos de tipo cualitativo, así como también revisiones o cartas al editor, textos no disponibles, estudios en contextos distintos al universitario o que no relacionaran estadísticamente al autocontrol y rendimiento académico.

En la primera selección se descartaron las investigaciones duplicadas (investigaciones presentes en dos o más bases de datos), después, para la segunda selección, cada autor realizó individualmente un proceso de examen de cada una de las investigaciones tomando en cuenta los criterios anteriormente mencionados. En el caso de artículos en que no quedaba clara su inclusión, los autores acordaron contabilizarlos inicialmente para después establecer un consenso sobre su inclusión o descarte, considerando los criterios establecidos inicialmente. En su conjunto, las investigaciones seleccionadas reportan las respuestas de 5.314 estudiantes, de ambos sexos, en su mayoría cursando los primeros años de formación, de diversas carreras universitarias, pertenecientes a 6 países (Alemania, Canadá, Arabia Saudita, Estados Unidos, Filipinas, Holanda). En la figura 1 se muestra un total de 173 trabajos, de los cuales solo 9 quedaron en la fase final de la revisión toda vez que eran investigaciones que analizaron la relación del autocontrol y el rendimiento académico en estudiantes universitarios.

Para el proceso de ordenamiento de los datos se empleó un protocolo de registro y organización de la información descrita de cada investigación, considerando autores, año, diseño del estudio, tamaño de la muestra, instrumentos de medida, medidas de fiabilidad, conceptualizaciones y correlación entre autocontrol y rendimiento académico. Para los diseños de investigación empleados, se utilizó la clasificación de diseños de investigación propuesta por Ato y Benavente (2013). Se realizó una descripción de las investigaciones seleccionadas considerando el tipo de diseño empleado. Para las conceptualizaciones de autocontrol y rendimiento académico, se registraron las definiciones conceptuales o referentes de las variables y los instrumentos de medida con sus características psicométricas. Grado de asociación de estas variables: se registró el grado de asociación ( $r$ ) de las variables y el nivel de significación. La detección de pocos estudios que respondieran a los objetivos planteados, así como la evidencia de estudios heterogéneos en términos de instrumentos de medición, y de distintos marcos metodológicos y conceptuales para estudiar el autocontrol, justifican el desarrollo de un revisión sistemática cualitativa sustentada en una descripción y evaluación de los estudios uno por uno, que indiquen sus diferencias y similitudes (Botella y Gambara, 2006). 


\section{RESULTADOS}

Se identificaron 173 artículos, de los cuales solo 9 cumplieron con los criterios de selección; 8 son de tipo predictivo-explicativo y uno es de tipo cuasi-experimental, la tabla 1 se muestra los estudios seleccionados de acuerdo a su tipo.

\section{Diseños empleados en el estudio del autocontrol y rendimiento académico.}

Las investigaciones seleccionadas son de carácter empírico, 8 emplearon diseño predictivo-explicativo. Los procedimientos de análisis estadísticos empleados fueron en su mayoría correlaciones, análisis de regresiones y modelo de mediación. Un trabajo de tipo cuasi-experimental se realizó por medio de mediciones de antes y después de la aplicación del programa en un solo grupo, en este caso el procedimiento de análisis estadístico fue correlaciones y regresiones lineales.

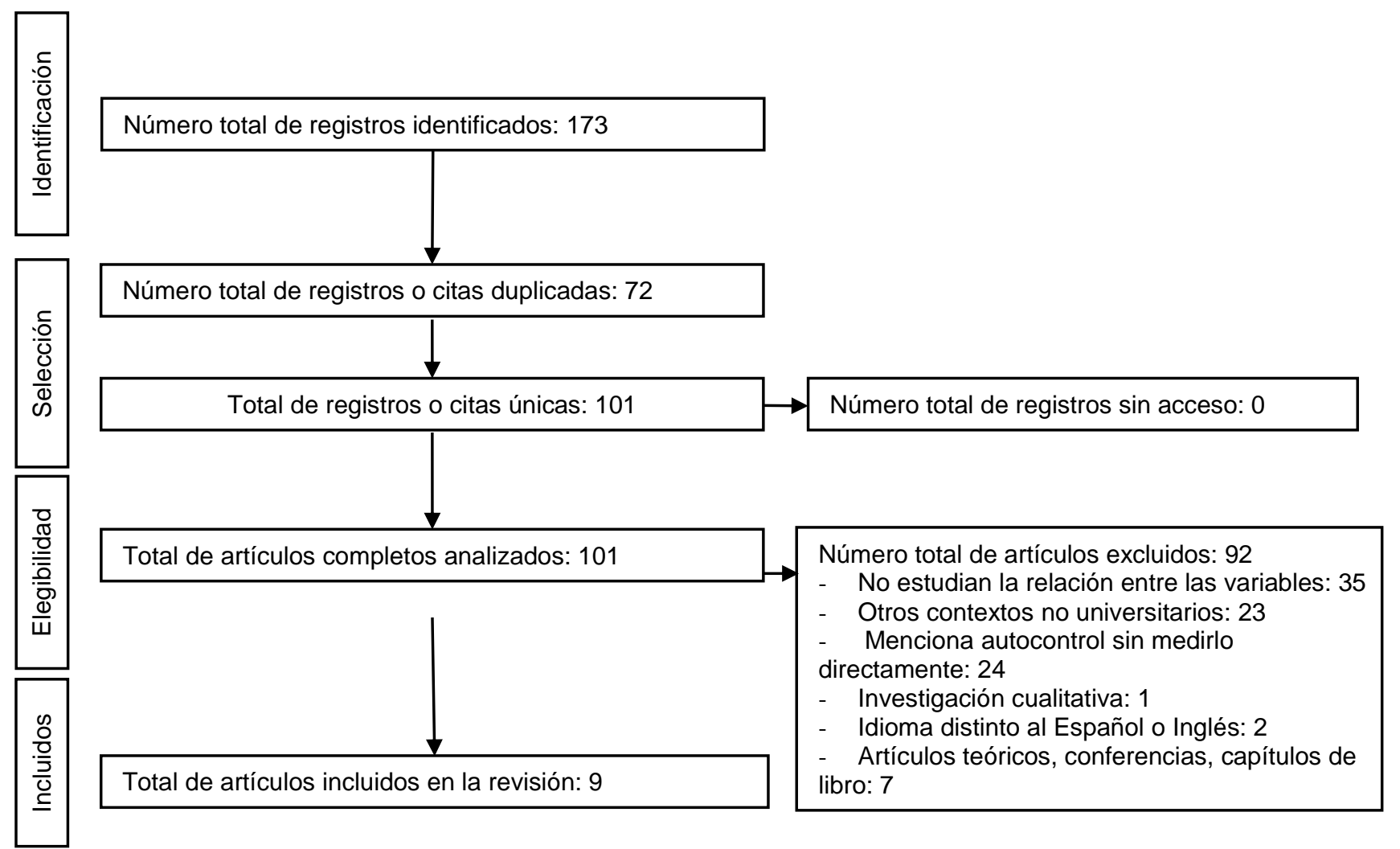

Fig. 1: Diagrama de flujo de la información de las fases de la revisión sistemática.

El estudio de Kennett y Reed (2009) tuvo como objetivo examinar los factores psicosociales que predicen el rendimiento y la retención en 33 estudiantes universitarios que asistieron a una intervención (curso) motivacional para el éxito. Se plantearon las hipótesis: (1) los estudiantes que tienden a emplear de forma menos efectiva sus habilidades conductuales y cognitivas (por ejemplo, alta ansiedad, mala autogestión académica y menor autoeficacia) se benefician de una intervención motivacional para el éxito, (2) los estudiantes que obtienen más altas calificaciones al final del año son académicamente más ingeniosos y muestran mayores niveles en el autocontrol académico después de asistir al curso motivacional, (3) las destrezas generales aprendidas (por ejemplo, autoeficacia) predicen la retención universitaria y (4) los estudiantes que tienen niveles más bajos de ansiedad se comportan mejor y son académicamente más ingeniosos. Se analizaron las respuestas de 33 estudiantes inscritos en un curso motivacional para el éxito en la universidad. Las conclusiones refieren efectos positivos del curso motivacional, sobre el desempeño y cómo puede ayudar a la transición del estudiante en la universidad.

En el trabajo presentado por Zettler (2011) se plantearon las hipótesis: (1) el autocontrol está más vinculado a los comportamientos de ciudadanía universitaria y (2) menos con los comportamientos académicos contraproducentes. Se presentan dos estudios, en el primero evaluaron las variables en dos momentos a 851 estudiantes de Psicología, en el segundo evaluaron una muestra de 223 estudiantes de la misma carrera. Se reporta una alta asociación entre autocontrol y comportamientos de ciudadanía universitaria, esta última vinculada a conductas que contribuyen a los objetivos de la formación universitaria, lo que beneficia al estudiante desde el punto de vista social y psicológico. Se reporta una relación inversa entre 
autocontrol y comportamiento académico contraproducente (por ejemplo, copiar en los exámenes).

El estudio de King y Gaerlan (2014) tuvo como objetivo examinar las emociones académicas e investigar la relación del autocontrol con las experiencias académicas de los estudiantes. Participaron 385 estudiantes universitarios filipinos. Presentan las hipótesis: (1) el autocontrol predice las emociones académicas y estas a su vez predicen el logro académico percibido, (2) el autocontrol es un antecedente de las emociones académicas positivamente valoradas (disfrute, esperanza y orgullo) y (3) el autocontrol predice directamente las emociones positivas e inversamente las emociones negativas. También evaluaron el efecto de mediación de las emociones en la relación entre el autocontrol, los resultados académicos, el compromiso y el logro. En sus resultados destacan el papel del autocontrol en la generación de emociones y el impacto que esto puede tener en el rendimiento académico del estudiante.

El estudio de Knouse et al. (2014) planteó los siguientes objetivos: (1) determinar si los déficits de funcionamiento ejecutivo predicen el rendimiento académico, (2) examinar las contribuciones únicas de cada una de las cinco dimensiones de las funciones ejecutivas en la predicción del rendimiento académico actual y futuro, (3) examinar la relación entre los déficit de funcionamiento cognitivo y la determinación de metas y (4) determinar si los objetivos de metas miden la relación entre los déficits en el funcionamiento cognitivo y desempeño académico. El autor realizó dos estudios en este trabajo, primero con una muestra de 314 y luego otra con 229 estudiantes. Se reporta que el déficit de funcionamiento ejecutivo se relaciona con el rendimiento académico, incluso cuando es controlado el efecto de las calificaciones anteriores de los estudiantes.

El estudio de Baars et al., (2015) evaluó la influencia de características de funcionamiento ejecutivo (niveles de atención, planificación y autocontrol) de los estudiantes sobre el rendimiento académico. Plantearon la hipótesis que las diferencias individuales en el desarrollo de habilidades neuropsicológicas (atención, planificación y autocontrol) pueden tener una influencia sustancial en el resultado del proceso educativo. El autocontrol fue considerado como parte de las funciones ejecutivas. Participaron 1332 estudiantes universitarios de primer año pertenecientes al área de ciencias aplicadas en dos oportunidades, durante la primera semana del semestre se evaluó funcionamiento ejecutivo, estilo de vida y, al finalizar el periodo académico, se obtuvo el rendimiento académico a partir de los exámenes. Los principales resultados mostraron que el nivel de autocontrol de los estudiantes es predictor de éxito en los estudios del primer año.

El estudio de Victoroff y Boyatzis (2013) tuvo como objetivo examinar la relación de las dimensiones de la inteligencia emocional en el desempeño de estudiantes de tercer y cuarto año de una escuela dental. Con una muestra de 79 estudiantes analizaron las variables de desempeño didáctico, preclínico, clínico y el rendimiento académico (capacidad cognitiva-perceptiva), en primero y segundo año académico. Otra investigación presentada, tuvo como objetivo evaluar la relación entre el aprendizaje autodirigido de los estudiantes y su desempeño académico, y evaluar el papel mediador de las percepciones de los estudiantes sobre el ambiente de aprendizaje (Alotaibi, 2016). La muestra estuvo constituida por 342 estudiantes de último año de la Carrera de Enfermería. Sus hipótesis fueron: (1) el nivel aprendizaje autodirigido de los estudiantes está positivamente relacionado con el rendimiento académico, (2) la percepción de los estudiantes sobre el entorno de aprendizaje media la relación entre el aprendizaje auto dirigido de los estudiantes y su desempeño académico. Se concluye que el nivel de aprendizaje autodirigido tiene relación positiva con el rendimiento académico, y las percepciones de los estudiantes sobre su entorno de aprendizaje desempeñaron un papel importante en esta relación.

El estudio de Stadler et al., (2016a) presenta la hipótesis que el autocontrol predice significativamente la varianza del logro (objetivo y subjetivo) incluso cuando la capacidad cognitiva es controlada. Buscó determinar si el autocontrol es importante para el rendimiento académico de los estudiantes más allá de su capacidad cognitiva general. Participaron 150 estudiantes universitarios de las carreras de Biología, Psicología y Ciencias del Deporte. Respondieron encuestas sobre habilidades cognitivas, autocontrol y percepción subjetiva de logro. Se registró el promedio de las calificaciones del semestre como medida objetiva del rendimiento académico. Se concluye que la capacidad cognitiva y de autocontrol son predictores del rendimiento académico. El autocontrol fue un predictor significativo del logro académico subjetivo $(r=.33, p<.001)$. Más recientemente, Honken, et al., (2016) desarrollaron una investigación con el objetivo de investigar la relación entre las calificaciones de los estudiantes de primer año de Ingeniería con el autocontrol, con la finalidad de identificar la contribución de esta variable en el desarrollo académico de los jóvenes. Participaron estudiantes de tres cohortes (2012, 2013 y 2014) de primer año de Ingeniería $(n=1295)$. Los resultados mostraron que el autocontrol explica el $4,2 \%$ de la varianza del rendimiento académico en el primer semestre, siendo relativamente consistente en las tres cohortes analizadas. El autocontrol puede manifestarse conductualmente en la gestión del estudio y, por tanto, influir positivamente en el éxito académico de los estudiantes de primer año. 


\section{Conceptualizaciones sobre autocontrol y rendimiento académico}

En la tabla 2 se presenta la descripción de las conceptualizaciones presentadas en las investigaciones analizadas. Los resultados refieren que no existe un consenso sobre la definición de autocontrol, ni de rendimiento académico. En el caso del autocontrol, se considera como una habilidad que se desarrolla y puede perfeccionarse a lo largo de la vida, que permite la autorregulación de conductas, emociones y pensamientos con la finalidad de lograr metas a largo plazo, puede ser vista como un constructo independiente y como parte de un conjunto de procesos psicológicos más complejos. En el caso del rendimiento académico podría afirmarse que este constructo es el resultado de conductas que permiten optimizar el desempeño y esfuerzo académico del estudiante de acuerdo a una serie de criterios académicos, que puede verse de forma específica, por ejemplo de una asignatura o a nivel general de todo el trabajo realizado a lo largo de un periodo académico, y que puede ser visto desde la mirada de sus docentes o desde la propia mirada del estudiante.

\section{Relaciones entre autocontrol y rendimiento académico}

Los resultados de la revisión descritos en la tabla 1, muestran que existe relación entre el autocontrol y el rendimiento académico (oscilando desde $r=.06$ a $r=.46$ ), siendo en su mayoría una relación media-alta significativa. Esta asociación varía de acuerdo a los tipos de mediciones empleadas. En la mayoria de los estudios analizados se encontraron altos niveles de correlación cuando el rendimiento académico se obtuvo por medio del reporte de las autoridades (calificaciones finales o promedios) o medidas objetivas (escalas de medición del desempeño académico) (Stadler et. al., 2016a; Zetler, 2011; Victoroff y Boyatzis, 2013; Kennett y Reed, 2009; Honken, et al., 2016). Cuando el autocontrol fue correlacionado con el rendimiento académico autodeclarado (calificación reportada por el estudiante) se encontraron resultados heterogéneos, en un caso presentó alta correlación (Alotaibi, 2016) y en otros casos se presentaron bajas correlaciones (King y Gaerlan, 2014; Knouse et al., 2014; Baars et al., 2015).

\section{DISCUSIÓN}

El análisis de los 9 trabajos publicados en las bases de datos Web of Science, Scopus, Scielo y Eric entre los años 2006 y 2017, buscó responder a los objetivos de (1) identificar los diseños de investigación más empleados en estudios sobre la relación entre autocontrol y rendimiento académico en estudiantes universitarios, (2) presentar las conceptualizaciones de autocontrol y rendimiento académico utilizadas en esos estudios y (3) identificar el grado de asociación encontrado entre estas variables. Tanto individualmente como en su conjunto, los trabajos constituyen en valiosos aportes a la comprensión de las relaciones entre el autocontrol y el rendimiento académico del estudiante en la vida universitaria.

1. A nivel de diseño, la mayoría de los estudios es de tipo predictivo-explicativo $(n=8 ; 88.88 \%)$, teniendo como objetivo explorar relaciones entre variables para pronosticar o explicar su comportamiento a partir de una muestra única de participantes. Usualmente se emplean medidas de naturaleza cuantitativa de dos o más variables, algunas son analizadas mediante correlaciones, regresiones y análisis de mediación. Entre las limitaciones de este tipo de diseño se encuentra la dificultad de identificar (a) los efectos de la eventual intervención de otras variables, como por ejemplo género, (b) la dirección de causalidad en las relaciones encontradas, (c) los tamaños de sus efectos y (c) la imposibilidad de manipular variables independientes (Ato y Benavente, 2013). Todos los estudios utilizaron medidas de autoinforme, es importante tomar en cuenta las limitaciones y aspectos objetables con el uso único de esta técnica en el proceso de recolección de información, basándose la investigación del fenómeno sólo en la percepción del estudiante. Tres de los estudios seleccionados no refieren información psicométrica de las medidas de autocontrol empleadas, el resto presentaron adecuados índices de consistencia interna (Streiner, et al., 2015). La mayoría de los estudios poseen muestras seleccionadas por conveniencia $(n=8 ; 88.88 \%)$, lo que limita la posibilidad de generalizar los resultados en otros contextos. Se han desarrollado en Asia ( $n=2 ; 22.22 \%)$, Europa $(n=3$; $33.33 \%)$ y América del Norte $(n=4 ; 44.44 \%)$. Resultados similares a los presentados en otras revisiones sistemáticas (de Ridder et al. 2012), donde la mayoría de los estudios se desarrollan en contextos como el americano o el europeo, en este escenario es de importancia efectuar investigación de estas variables en la vida universitaria latinoamericana y publicarlas en bases de datos con revistas de alto impacto como las consideradas en la presente revisión.

En cuanto a la medición, se presentó el uso de varias escalas, aun cuando algunas investigaciones ( $n=3$; 33.33\%) emplearon la Escala de autocontrol de Tangney et al. (2004) se presentaron limitaciones en la comparación de los resultados presentados, impidiendo el establecimiento de comparaciones en los niveles de autocontrol de los estudiantes en los distintos estudios. Esto pone de manifiesto las dificultades en el estudio del autocontrol, debido a la gran cantidad de medidas de auto informe presentes en la literatura 
científica (Duckworth y Kern, 2011). El disponerse de un único estudio desarrollado bajo condiciones experimentales, limita las evidencias sobre la relación causal existente entre el autocontrol y el rendimiento académico. Esto manifiesta las dificultades de este tipo de estudios, asociada a disponer de los instrumentos de medición para la aplicación antes y después del tratamiento, la necesidad de participantes suficientes para cada condición del estudio (experimental/control), y la creación de nuevos programas de intervención. Hay desafíos en estos aspectos para estudiar el impacto o efecto del autocontrol en el rendimiento académico de los estudiantes universitarios.

2. A nivel conceptual, los trabajos analizados muestran desacuerdos sobre las concepción del autocontrol, presentándose diversidades en su definición, por ejemplo, ha sido descrito como una habilidad o capacidad (Kennett y Reed, 2009; King y Gaerlan, 2014; Stadler, Aust, et al., 2016a), como dimensión de otras variables como la inteligencia emocional (Victoroff y Boyatzis, 2013) o como parte de las funciones ejecutivas (Baars et al., 2015; Knouse et al., 2014; Zettler, 2011). La diversidad de conceptos favorece su investigación en diversos aspectos del comportamiento humano. Las distintas visiones del autocontrol se presentan debido a que está relacionado con una amplia gama de comportamientos, complejizando el establecimiento de acuerdos respecto a su definición, generando una amplia variedad de marcos teóricos y metodológicos para su estudio y operacionalización (Duckworth y Kern, 2011). Esto dificulta la integración, medición y análisis de los hallazgos y su aporte especifico en el contexto educativo. Sin embargo, es claro es que el autocontrol incluye la regulación exitosa de los impulsos, siendo una característica de la conducta de autorregulación y un rasgo opuesto a impulsividad (Duckworth y Kern, 2011; Tangney et al., 2004).

Autocontrol puede ser definido como (1) una capacidad que transita por un proceso de maduración durante el crecimiento, haciendo relevante la identificación temprana de los niveles de su desarrollo en los estudiantes y, por tanto, (2) es una habilidad que puede ser entrada para su perfeccionamiento, (3) las investigaciones muestran la existencia de una relación de magnitud de baja-media, significativa, entre el autocontrol y el rendimiento académico en los estudiantes universitarios. (4) El autocontrol permite el desarrollo de comportamientos beneficiosos y fomenta la presencia de interacciones sociales saludables (Kennett y Reed, 2009; Knouse et al., 2014), (5) favorece el desempeño objetivo y subjetivo del rendimiento (Stadler, Aust, et al., 2016a; Zettler, 2011). (6) Es una variable que está inversamente vinculada a conductas contraproducentes (Zettler, 2011) siendo altamente relevante en la prevención del abandono académico.

3. Pocos estudios que relacionan estas variables y los resultados de una asociación heterogénea se presentan de forma similar a lo reportado en la revisión de Kim y Seo (2015), se diferencian en que la mayoría presentan correlaciones significativas, confirmando lo que esperábamos al inicio del presente estudio. La diferencia en los resultados podría deberse al uso de distintas escalas de medida de autocontrol y el empleo de distintos indicadores de rendimiento académico. Otro aspecto importante para la comprensión del autocontrol en este contexto podría ser la presencia de variables moderadoras que tengan efectos en esta relación, sin embargo, en los estudios analizados no queda claro la identificación de estas variables debido a que los estudios no consideran diferencias entre el género, carrera, semestre u otra característica que pudiera jugar este papel moderador.

La presencia de investigaciones con intervenciones, así como la evidencia de los efectos positivos en el rendimiento académico (Kennett \& Reed, 2009), resalta la importancia de considerar en los entrenamientos o cursos introductorios a los estudiantes al momento de iniciar las actividades académicas universitarias. A nivel individual se destaca el desarrollo de la habilidad de autocontrol para contribuir no solo en el rendimiento, sino también en el incremento del bienestar y la salud mental durante el desarrollo de la vida universitaria (de Ridder et al., 2012).

Mucho se ha investigado sobre el rendimiento académico en los estudiantes universitarios, pero son menos los trabajos que lo relacionan con el autocontrol. Aparece la conveniencia de efectuar más investigaciones con poblaciones estudiantiles, en diversas regiones, con muestras amplias, de distintos niveles educacionales, orientados a examinar el rol del autocontrol en el rendimiento. Además, considerando estrategias adicionales para su estudio, tales como uso de tareas combinadas con medidas de autoinforme. En el caso del rendimiento académico es importante considerar medidas de autorreporte y otras formas de medición. Resultaría interesante estudiar los efectos de moderación de variables como la edad, el género, carrera, y de mediación con características de personalidad, empleo de estrategias de autorregulación, perfeccionismo, procrastinación y compromiso, y usar diseños de tipo experimental para profundizar la comprensión de los efectos de esta variable. 


\begin{tabular}{|c|c|c|c|c|}
\hline 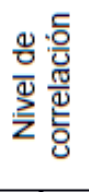 & 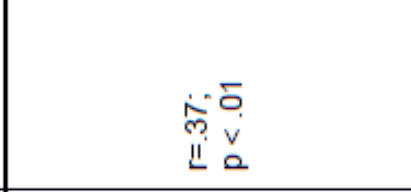 & 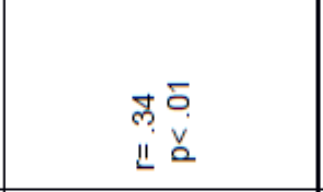 & 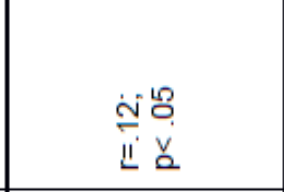 & 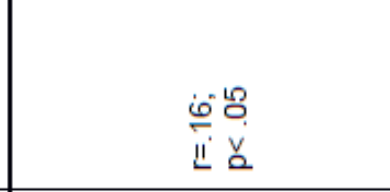 \\
\hline 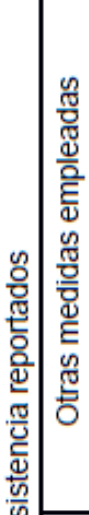 & 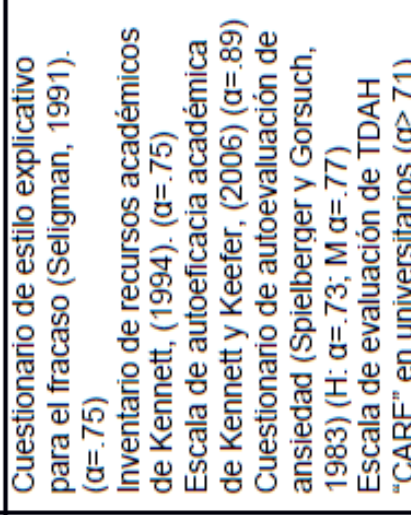 & 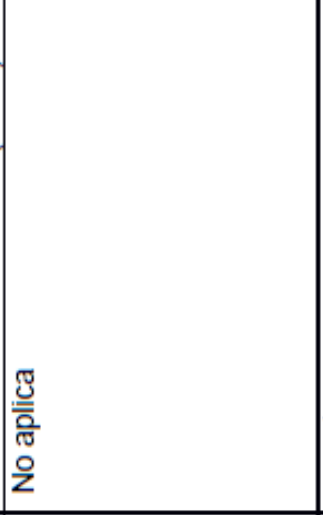 & 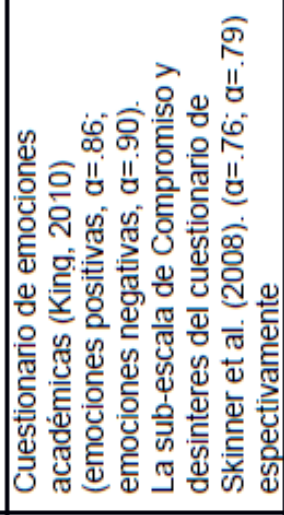 & 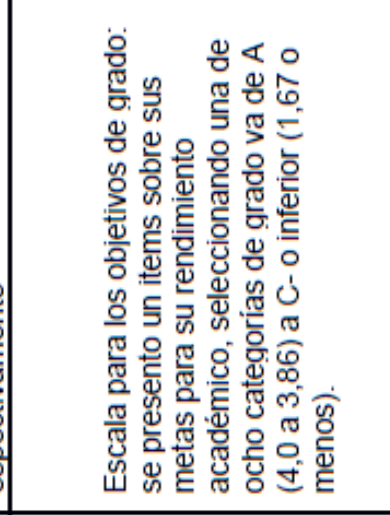 \\
\hline 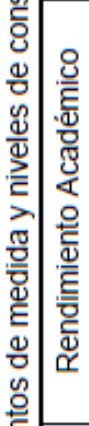 & 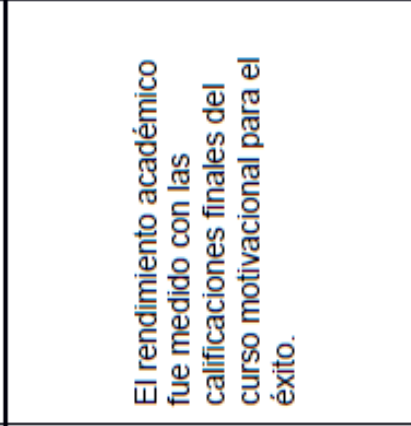 & 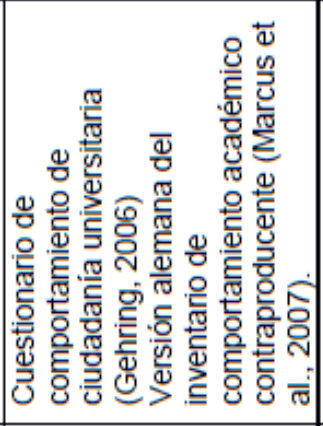 & 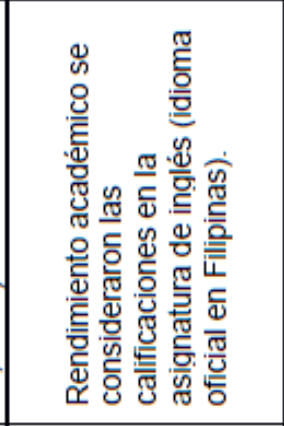 & 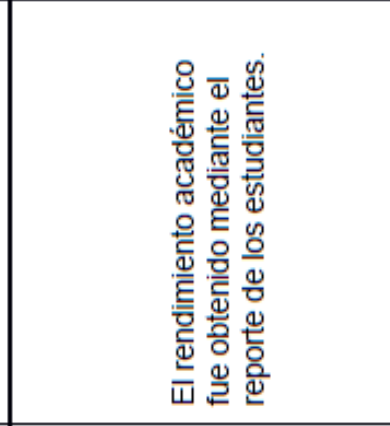 \\
\hline 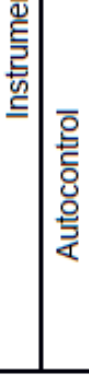 & 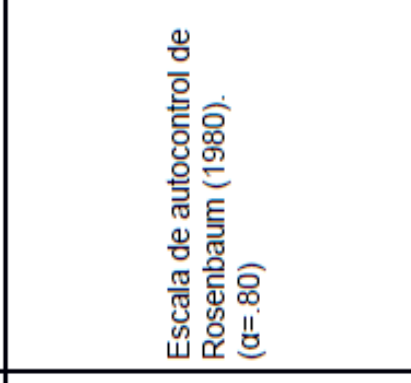 & 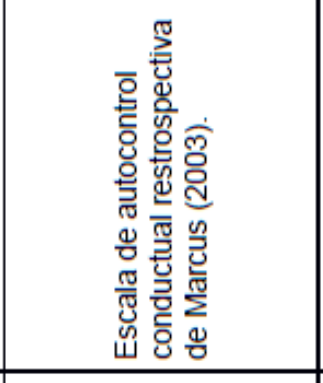 & 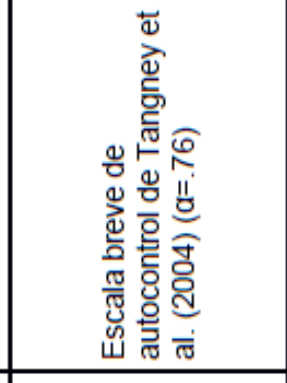 & 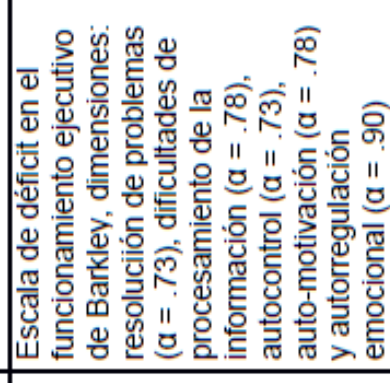 \\
\hline 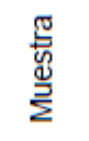 & m & 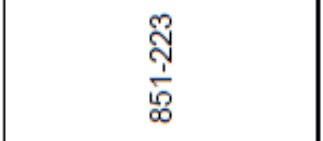 & $\stackrel{\mathscr{L}}{\infty}$ & 㩊 \\
\hline 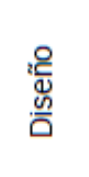 & 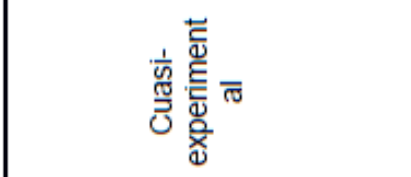 & 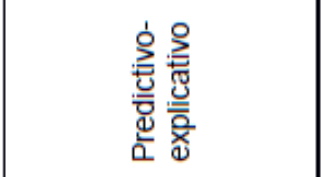 & 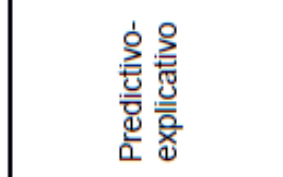 & 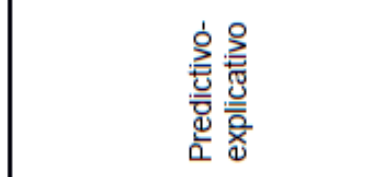 \\
\hline 을 & 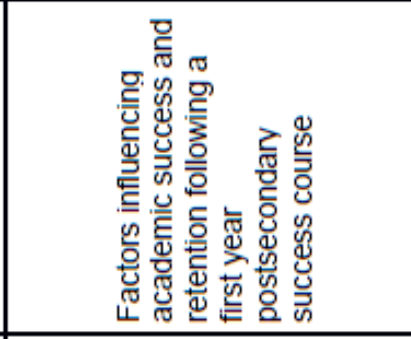 & 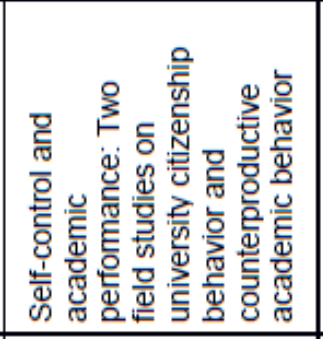 & 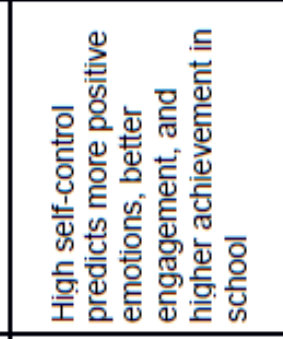 & 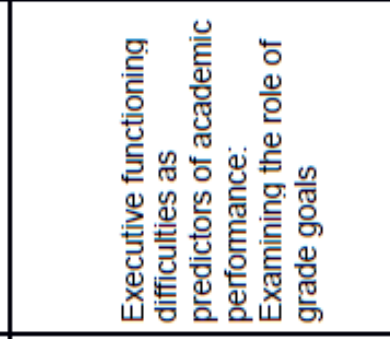 \\
\hline 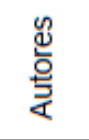 & 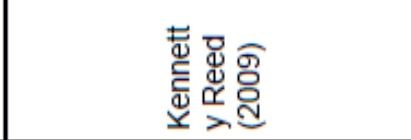 & 离急 & 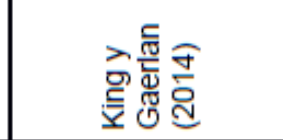 & 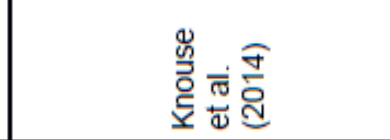 \\
\hline$z$ & - & $N$ & $m$ & $\checkmark$ \\
\hline
\end{tabular}




\begin{tabular}{|c|c|c|c|c|c|}
\hline 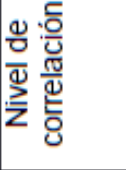 & 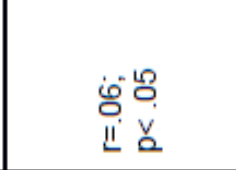 & 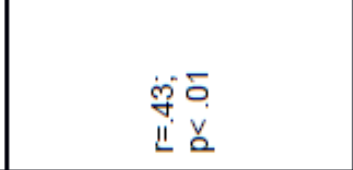 & $\begin{array}{l}\dot{\varphi} \bar{c} \\
\text { II v } \\
\text { II }\end{array}$ & 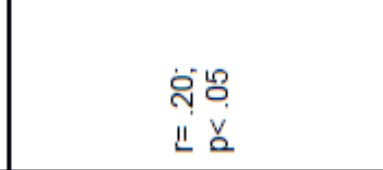 & 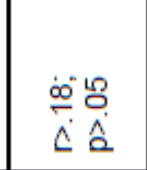 \\
\hline \multirow{3}{*}{ 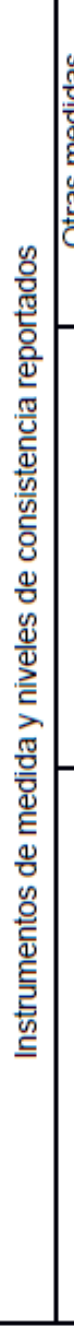 } & 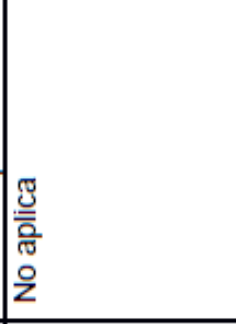 & 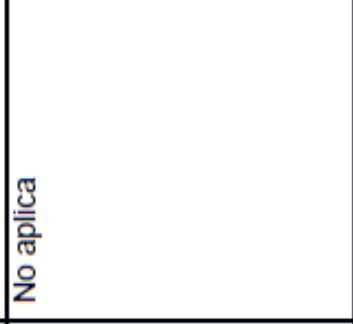 & 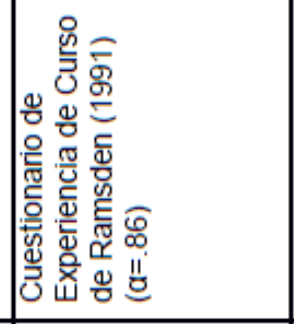 & 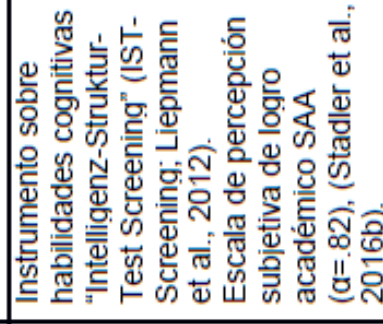 & 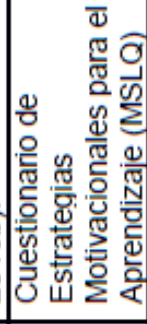 \\
\hline & 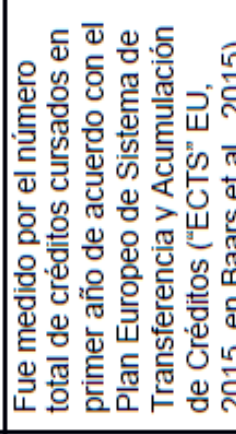 & 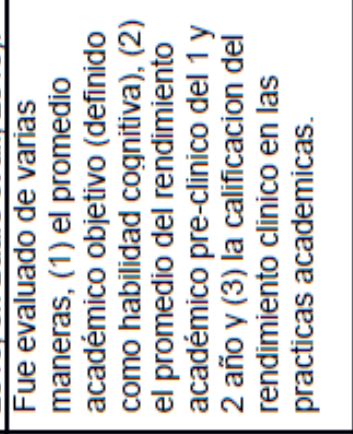 & 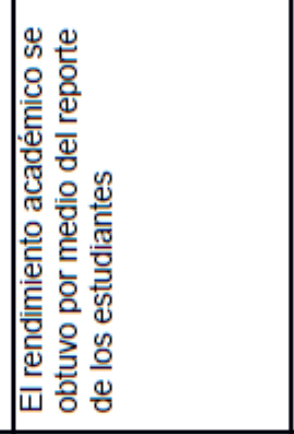 & 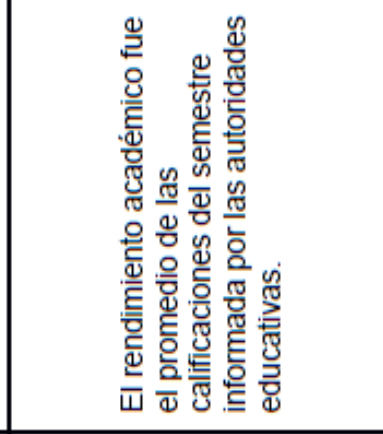 & 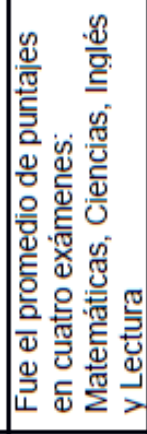 \\
\hline & 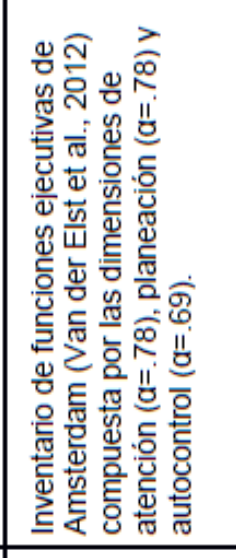 & 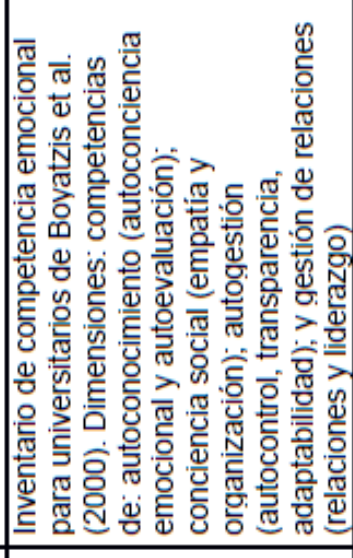 & 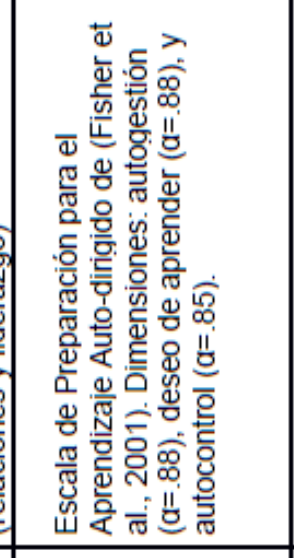 & 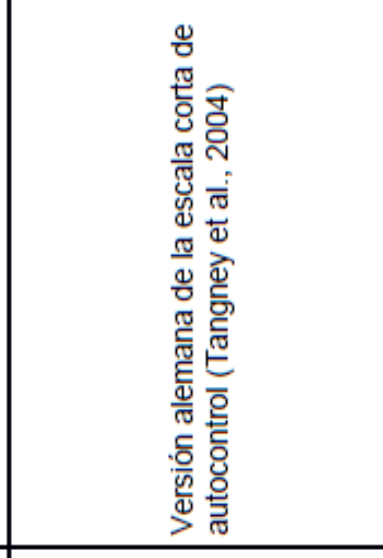 & 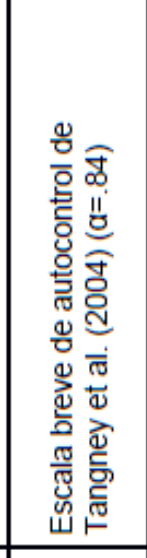 \\
\hline 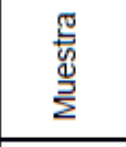 & ్ָల్ల & $\stackrel{\mathscr{0}}{\leftarrow}$ & సै & $\stackrel{\circ}{\circ}$ & 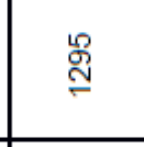 \\
\hline 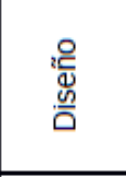 & 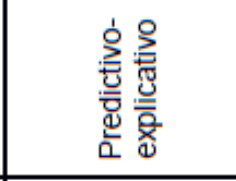 & 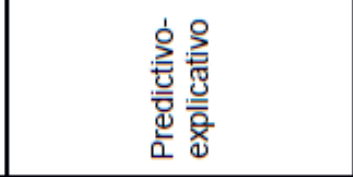 & 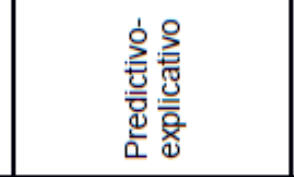 & 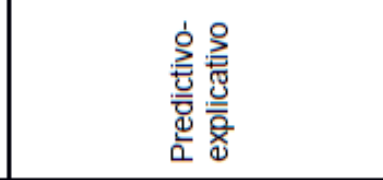 & 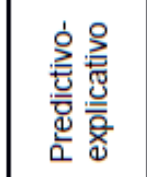 \\
\hline 凝 & 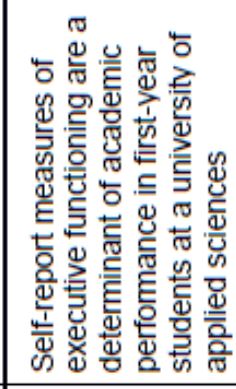 & 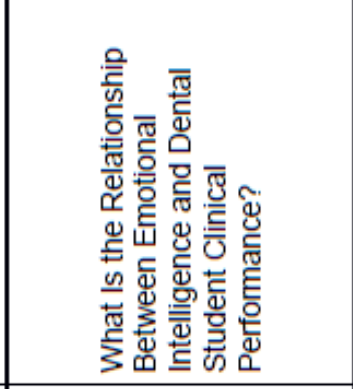 & 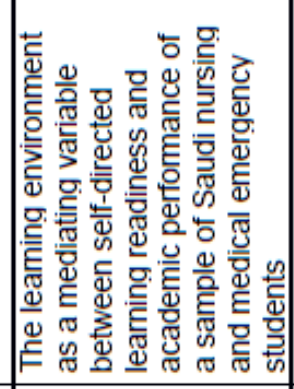 & 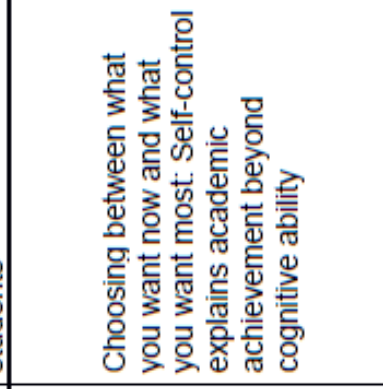 & 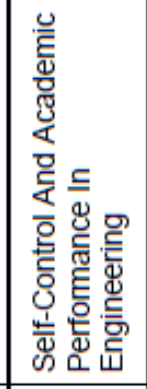 \\
\hline 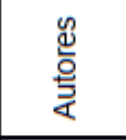 & 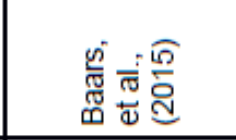 & 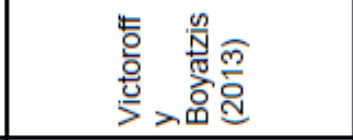 & 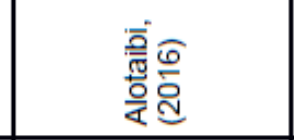 & 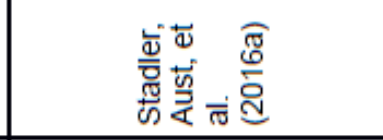 & 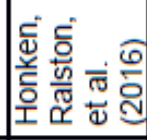 \\
\hline z & 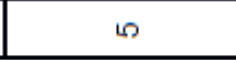 & 0 & $\Lambda$ & $\infty$ & $\sigma$ \\
\hline
\end{tabular}




\begin{tabular}{|c|c|c|c|c|c|c|c|c|c|}
\hline 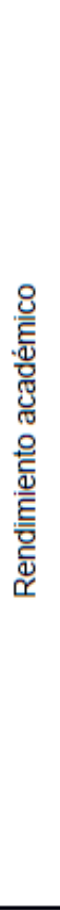 & 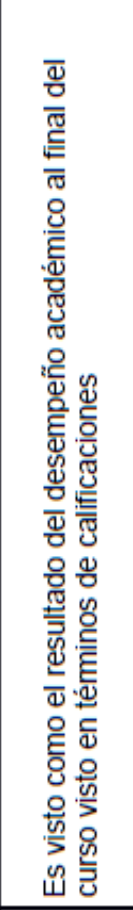 & 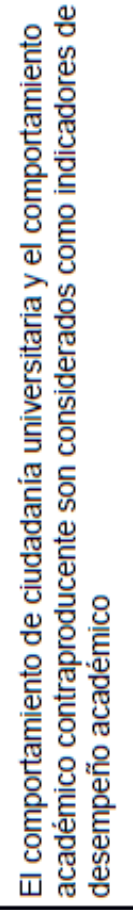 & 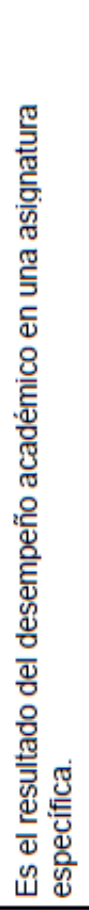 & 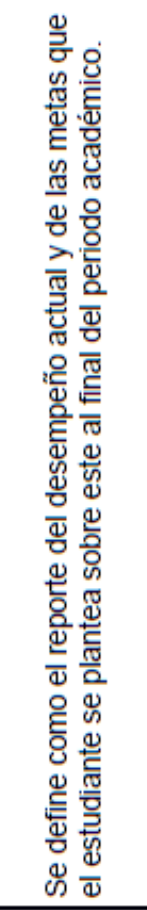 & 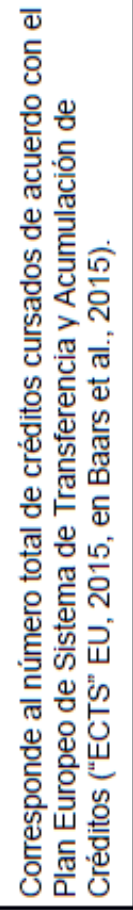 & 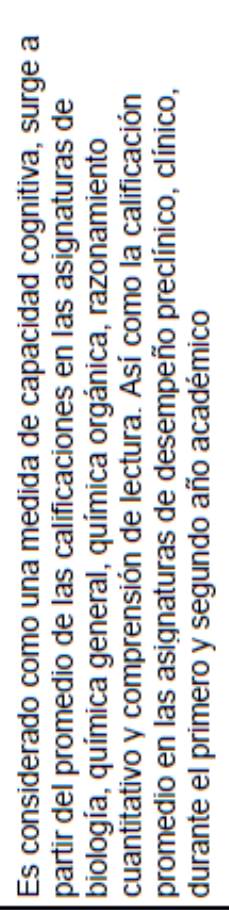 & 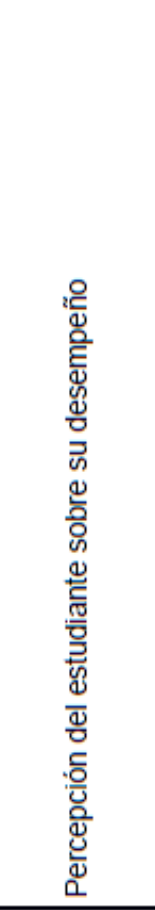 & 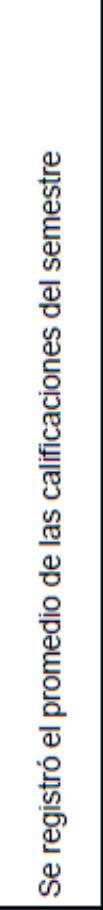 & 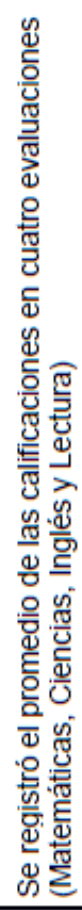 \\
\hline 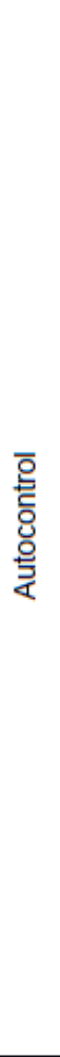 & 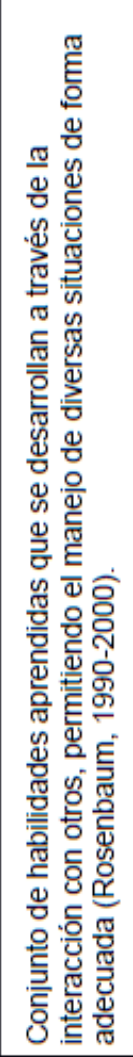 & 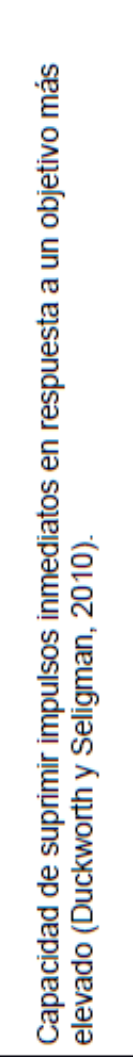 & 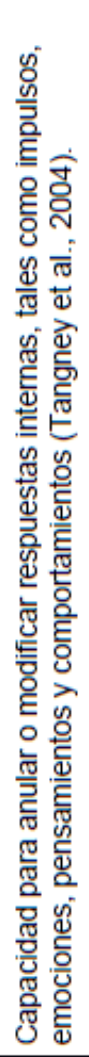 & 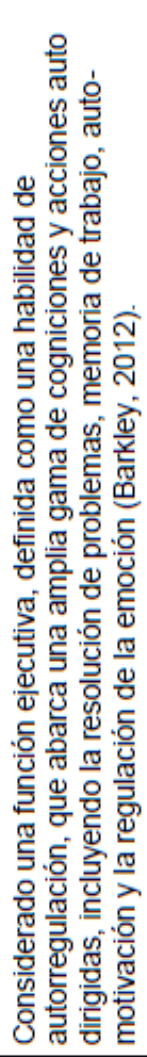 & 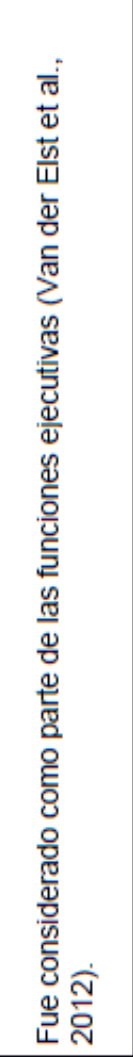 & 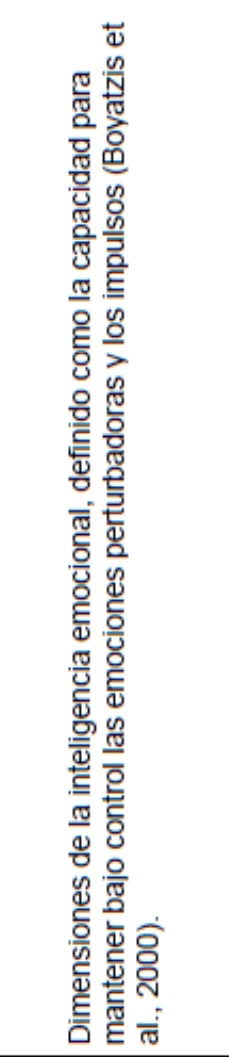 & 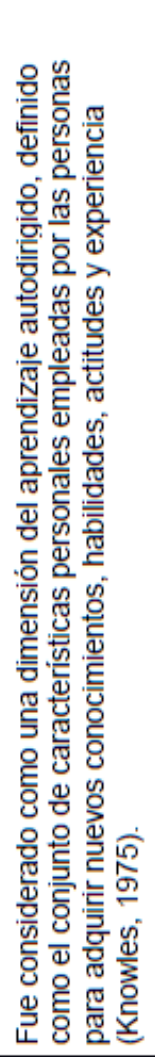 & 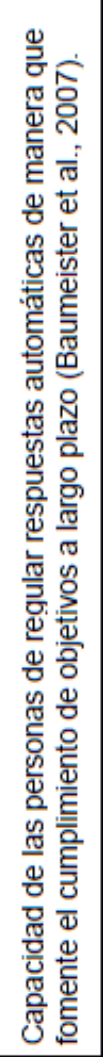 & 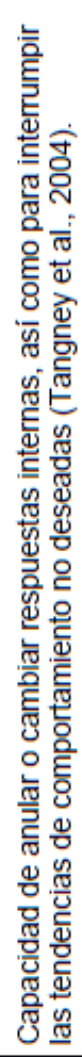 \\
\hline $\begin{array}{l}\frac{\mathscr{3}}{0} \\
\frac{0}{3}\end{array}$ & 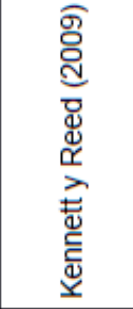 & 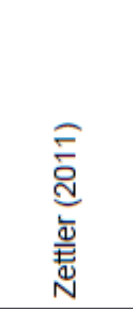 & 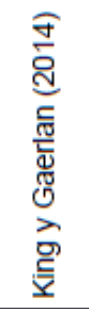 & 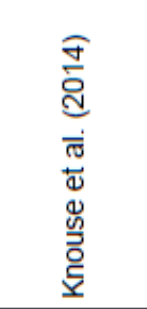 & 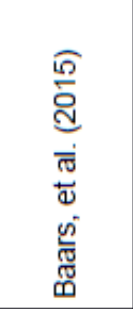 & 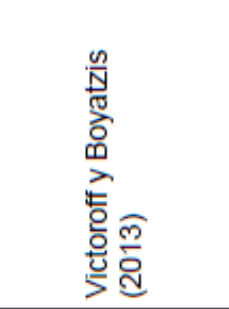 & 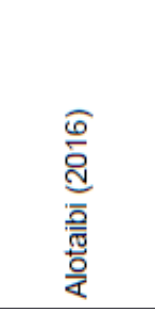 & 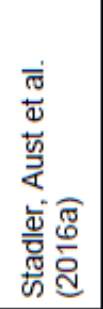 & 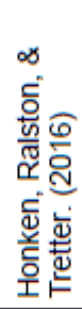 \\
\hline$z$ & - & $N$ & $m$ & $\nabla$ & م & 0 & $r$ & $\infty$ & $\sigma$ \\
\hline
\end{tabular}




\section{CONCLUSIONES}

De acuerdo a los resultados y discusión del presente estudio, se pueden extraer las siguiente conclusiones principales, 1) la investigación sobre las relaciones entre autocontrol y rendimiento académico en su mayoría tienen un diseño de tipo predictivo-explicativo, 2) se presentan correlaciones heterogéneas entre estas variables, 3) el autocontrol es un constructo amplio que incluye la regulación exitosa de impulsos, vinculado a diversos comportamientos, es una habilidad que permite la autorregulación de conductas, emociones y pensamientos con la finalidad de lograr metas a largo plazo, que van desde la generación de conductas saludables como la asistencia a clases, desarrollo de interacciones sociales, y manejo de emociones hasta un alto desempeño, 4) transita por un proceso de maduración durante el crecimiento y desarrollo de los estudiantes, 5) el rendimiento académico es el resultado de conductas que permiten optimizar el desempeño y esfuerzo del estudiante. 6) Los estudios analizados han sido desarrollados en contextos distintos y con muestras por conveniencia, empleando medidas de auto informe para la medición del autocontrol y una variedad de medidas para el rendimiento académico.

\section{AGRADECIMIENTO}

Este trabajo ha sido financiado por el Proyecto Fondecyt 1161502: "Modelo explicativo de la permanencia y el abandono de los estudios universitarios, basado en procesos cognitivos motivacionales"

\section{REFERENCIAS}

Alotaibi, K. N., The Learning Environment as a mediating variable between Self-directed learning readiness and Academic Performance of a sample of saudi nursing and medical emergency students, doi: https://doi.org/10.1016/j.nedt.2015.11.003, Nurse Education Today, 36, 249-254 (2016)

Ato, M., López, J. J., y A. Benavente, Un sistema de clasificación de los Diseños de Investigación en psicología, doi: dx.doi.org/10.6018/analesps.29.3.178511, Anales de Psicología, 29(3), 1038-1059 (2013)

Avendaño, C., Gutiérrez, K.A., Salgado, C.F., y M.A. Dos-Santos, Rendimiento Académico en Estudiantes de Ingeniería Comercial: Modelo por Competencias y Factores de Influencia, doi: http://dx.doi.org/10.4067/S071850062016000300002, Formación Universitaria, 9(3), 03-10 (2016)

Baars, M.A., M.N. Bijvank, G.H., Tonnaer y J. Jolles, Self-report measures of Executive Functioning are a determinant of Academic Performance in first-year Students at a University of Applied Sciences, doi:10.3389/fpsyg.2015.01131, Frontiers in Psychology, 6 (1131), 1-7 (2015)

Barkley, R. A., Executive functions: What they are, how they work, and why they evolved. Guilford Press, New York, United States (2012)

Baumeister, R.F., K.D. Vohs y D.M. Tice, The strength Model of Self-control, doi: 10.1111/j.1467-8721.2007.00534.x, Current Directions in Psychological Science, 16(6), 351-355 (2007)

Baumeister, R.F., y J.J. Exline, Virtue, Personality, and Social Relations: Self-control as the moral muscle, doi: 10.1111/1467-6494.00086, Journal of Personality, 67(6), 1165-1194 (1999)

Baumeister, R.F., y J.J. Exline, Self-control, Morality, and Human Strength. Journal of Social and Clinical Psychology, doi: https://doi.org/10.1521/jscp.2000.19.1.29, 19(1), 29-42 (2000)

Baumeister, R.F., Heatherton, T.F., y D.M. Tice, Losing control: How and why people fail at Self-regulation, $1^{\mathrm{a}}$ Ed., 6256. Academic Press, San Diego, Estados Unidos (1994)

Bertrams, A., Baumeister, R.F., y C. Englert, Higher Self-control capacity predicts lower Anxiety-impaired cognition during math examinations, doi: 10.3389/fpsyg.2016.00485, Frontiers in psychology, 7, 1-10 (2016)

Botella, J., Gambara, H., Doing and reporting a Meta-analysis, https://goo.gl/anQAku; ISSN 1697-2600, International Journal of Clinical and Health Psychology 6 (2), 425-440 (2006)

Burnette, J. L., E. K. Davisson, E. J. Finkel, D. R. V. Tongeren, C. M. Hui y R. H. Hoyle, Self-Control and Forgiveness, doi: https://doi.org/10.1177/1948550613502991, Social Psychological and Personality Science, 5(4), 443-450 (2014)

Conley, C.S., Kirsch, A.C., Dickson, D.A., y F.B. Bryant, Negotiating the Transition to College, doi: 10.1177/2167696814521808, Emerging Adulthood, 2(3), 195-210 (2014)

De Ridder, D. T., G. Lensvelt-Mulders, C. Finkenauer, F. M. Stok, y R. F. Baumeister, Taking stock of self-control: A Metaanalysis of how trait Self-control relates to a wide range of behaviors, doi: https://doi.org/10.1177/1088868311418749, Personality and Social Psychology Review, 16(1), 76-99 (2012) 
Duckworth, A.L., y M.L.A. Kern, Meta-analysis of the convergent validity of Self-control measures, doi: https://doi.org/10.1016/j.jrp.2011.02.004, Journal of Research in Personality, 45(3), 259-268 (2011)

Duckworth, A. L., E, Tsukayama, y H. May, Establishing causality using longitudinal hierarchical linear modeling: An illustration predicting Achievement from Self-control, doi: https://doi.org/10.1177/1948550609359707, Social psychological and personality science, 1(4), 311-317 (2010).

Eckberg, N., A. M. Pidgeon y H. Magyar, Examining the Psychosocial and Academic Factors Predicting Depression and Anxiety Symptomology Across First-Year and Later-Year University Students, doi:10.19044/esj.2017.v13n17p1, ESJ, 13(17), 1-16 (2017)

Finkenauer, C., R. Engels, y R. Baumeister, Parenting Behaviour and Adolescent Behavioural and eEmotional problems: The role of Self-control, doi: 10.1080/01650250444000333, International Journal of Behavioral Development, 29(1), 5869 (2005)

Hofmann, W., M. Luhmann, R.R. Fisher, K.D. Vohs, y R.F. Baumeister, yes, but are they happy? Effects of trait Self-control on affective Well-being and Life Satisfaction, doi: 10.1111/jopy.12050, Journal of Personality, 82(4), $265-277$ (2014)

Hofmann, W., Baumeister, R.F., Förster, G., y K.D. Vohs, Everyday temptations: an experience sampling study of Desire, Conflict, and Self-control, doi: 10.1037/a0026545, Journal of personality and social psychology, 102(6), 1318 (2012)

Honken, N., Ralston, P.A., y T.R. Tretter, Self-Control and Academic Performance in Engineering, doi: https://doi.org/10.19030/ajee.v7i2.9831, American Journal of Engineering Education, 7(2), 47 (2016)

Kennett, D. J., y M. J. Reed, Factors influencing Academic Success and Retention following a 1st-year post-secondary success course, doi: http://dx.doi.org/10.1080/13803610902804382, Educational Research and Evaluation, 15(2), 153166 (2009)

Kim, K. R., y E. H. Seo, The Relationship between Procrastination and Academic Performance: A Meta-analysis, doi: https://doi.org/10.1016/j.paid.2015.02.038, Personality and Individual Differences, 82, 26-33 (2015)

King, R.B., y M.J.M. Gaerlan, High Self-control Predicts more Positive Emotions, Better Engagement, and Higher Achievement in school, doi: 10.1007/s10212-013-0188-z, European journal of psychology of education, 29(1), 81-100 (2014)

Knouse, L.E., G. Feldman y E.J. Blevins, Executive functioning difficulties as predictors of Academic Performance: examining the role of Grade Goals, doi: https://doi.org/10.1016/j.lindif.2014.07.001, Learning and Individual Differences, $36,19-26(2014)$

Páramo, P., y C. Hederich, Educación Basada en la Evidencia, https://goo.gl/qVnbyJ, ISSN 0120-3916, Revista Colombiana de Educación, (66), 13-16 (2014)

Pérez-Cárceles, M. C., Gómez-Gallego, M., Gómez-Gallego, J. C., Palazón-Pérez de Los Cobos, A., y J. Gómez-García, El género como variable moderadora de los Resultados Académicos en la Enseñanza Universitaria, https://goo.gl/6fWXbH, ISSN 1578-4460, Regional and Sectoral Economic Studies, 14(3), 55-64 (2014)

Piquero, A. R., W. G. Jennings, D. P. Farrington, B. Diamond, M. Jennifer y R. A. Gonzalez, meta-analysis update on the effectiveness of early Self-control improvement programs to improve self-control and reduce delinquency, doi: 10.1007/s11292-016-9257-z, Journal of Experimental Criminology, 12(2), 249 (2016)

Rosenbaum, M. A., Schedule for assessing Self-control Behaviors: Preliminary findings, doi: https://doi.org/10.1016/S00057894(80)80040-2, Behavior therapy, 11(1), 109-121 (1980).

Sánchez-Meca, J., y J. Botella, Revisiones sistemáticas y meta-análisis: Herramientas para la práctica profesional, http://www.redalyc.org/articulo.oa?id=77812441002, ISSN 0214-7823, Papeles del Psicólogo, 31(1) 7-17 (2010)

Soria-Barreto, K., y S. Zúñiga-Jara, Aspectos determinantes del éxito Académico de Estudiantes Universitarios, doi: http://dx.doi.org/10.4067/S0718-50062014000500006, Formación universitaria, 7(5), 41-50 (2014)

Stadler, M., M. Aust, N. Becker, C. Niepel, y S. Greiff, Choosing between what you want now and what you want most: Self-control explains Academic Achievement beyond Cognitive Ability, doi: https://doi.org/10.1016/j.paid.2016.01.029, Personality and Individual Differences, 94, 168-172 (2016a)

Stadler, M. J., N. Becker, S. Greiff, y F. M. Spinath, The complex route to success: complex problem-solving skills in the Prediction of University Success, doi: http://dx.doi.org/10.1080/07294360.2015.1087387, Higher Education Research y Development, 35(2), 365-379 (2016b)

Streiner, D.L., Norman, G.R., y J. Cairney, Health Measurement Scales: a Practical Guide to their development and use, 5ta edición, 1-415. Oxford University Press, Oxoford, Inglaterra (2015)

Tangney, J.P., R.F. Baumeister y A.L. Boone, High Self-control predicts good Adjustment, less Pathology, Better grades, and interpersonal success, doi: 10.1111/j.0022-3506.2004.00263.x, Journal of personality, 72(2), 271-324 (2004) 
Tarbetsky, A. L., A. J. Martin, y R. J. Collie, Social and Emotional Learning, Social and Emotional Competence, and Students' Academic Outcomes: The Roles of Psychological Need Satisfaction, Adaptability, and Buoyancy. In E. Frydenberg, A. J. Martin y R. J. Collie (Eds.), Social and Emotional Learning in Australia and the Asia-Pacific: Perspectives, Programs and Approaches (pp. 17-37). Singapore: Springer Singapore (2017)

Uziel, L., y R. F. Baumeister, The Self-Control Irony: Desire for Self-Control Limits Exertion of Self-Control in Demanding Settings, doi: 10.1177/0146167217695555, Personality and Social Psychology Bulletin, 43(5), 693-705 (2017)

Van der Elst, W., C. Ouwehand, G. Van der Werf, H. Kuyper, N. Lee y J. Jolles, The Amsterdam Executive Function Inventory (AEFI): psychometric properties and demographically corrected normative data for adolescents aged between 15 and 18 years, doi: 10.1080/13803395.2011.625353, J. Clin. Exp. Neuropsychol, 34(2), 160-171 (2012)

Valiente, C., N. Eisenberg, T. L. Spinrad, R. Haugen, R, M. S. Thompson y A. Kupfer; Effortful Control and Impulsivity as Concurrent and Longitudinal Predictors of Academic Achievement, doi: 10.1177/0272431613477239, The Journal of Early Adolescence, 33(7), 946-972 (2013)

Vazsonyi, A. T., Mikuška, J., y E. L. Kelley, It's time: A meta-analysis on the Self-control-deviance link, doi: https://doi.org/10.1016/j.jcrimjus.2016.10.001, Journal of Criminal Justice, 48, 48-63 (2017)

Victoroff, K. Z., y E.R. Boyatzis, What is the relationship between Emotional Intelligence and Dental Student clinical performance?, http://www.jdentaled.org/content/77/4/416.long, ISSN: 1930-7837, Journal of dental education, 77(4), 416426, (2013)

Vidal, M., Oramas, J. y R. Borroto, Revisiones Sistemáticas. Educación Médica Superior, https://goo.gl/4YEo8v, ISSN 1561-2902, Educación Media Superior, 29(1), 198-207 (2015)

Wiese CW, Tay L, Duckworth A, et al, Too much of a good thing? Exploring the inverted-U relationship between Selfcontrol and Happiness, doi: 10.1111/jopy.12322, Journal of Personality, 2017, 1-17 (2017)

Zettler, I., Self-control and Academic Performance: Two field studies on university citizenship behavior and counterproductive Academic Behavior, doi: 10.1016/j.lindif.2010.11.002, Learning and Individual Differences, (en línea), 21(1), 119-123 (2011) 